\title{
Nonlinear Simulation of Wave Train Impact on a Vertical Seawall
}

\author{
Dezhi Ning, Xiang Li and Chongwei Zhang * iD \\ State Key Laboratory of Coastal and Offshore Engineering, Dalian University of Technology, \\ Dalian 116024, China; dzning@dlut.edu.cn (D.N.); xiangli@mail.dlut.edu.cn (X.L.) \\ * Correspondence: chongweizhang@dlut.edu.cn; Tel.: +86-0411-8470-8520 (ext. 8403)
}

Received: 24 June 2018; Accepted: 24 July 2018; Published: 26 July 2018

\begin{abstract}
A 2D nonlinear numerical wave flume is developed to investigate the wave train impact on a vertical seawall. Fully nonlinear kinematic and dynamic boundary conditions are satisfied on the instantaneous free surface. Cases of single-, double- and multi-crest wave trains are discussed. For single-crest wave train cases, the present nonlinear results are compared with the solution of the Serre-Green-Naghdi (SGN) model, showing good agreement. For double-crest wave train cases, the SGN model underestimates the maximum wave run-up along the vertical seawall. Compared with the linear results, the nonlinearity for double-crest cases can lead to an evident increase of the wave run-up and high-frequency free-surface oscillations. Through a fast Fourier analysis, evident nonlinear characteristics of the time series of the wave run-up and wave load during the wave impact process are confirmed. For multi-crest wave train cases, irregular wave run-ups can be observed. In some cases, the wave run-up along the vertical seawall can reach about 6 times that of the incident wave, which should be considered carefully in a practical design.
\end{abstract}

Keywords: nonlinear wave; wave run-up; wave impact; BEM; coastal structure

\section{Introduction}

Vertical seawalls are commonly used for coastal protection. In rough sea conditions where extreme waves exist, seawall is periodically impacted by shoaling waves, accompanied by high wave run-ups and hydrodynamic loads. Therefore, a systematic understanding of this interaction mechanism under extreme conditions is of great importance to guarantee structural safety. The extreme conditions may be caused by freak waves or rogue waves, and its interaction with structures has been widely studied during the past decades [1-3]. Recently, a new type of wave has attracted people's attention. An investigation into tsunamis reveals the fact that the tsunami wave may disintegrate into a wave train with smaller wave length, after its long-time propagation [4], and the number of wave crests inside the wave train is finite. High wave run-up can be observed when the wave train interacts with structures, and little research has been done on this. Even so, this wave train can still be simplified as continuous regular waves when the number of waves is infinite, or a solitary wave when there is only one wave crest inside the wave train; both of them have been studied extensively.

On regular wave interaction with a vertical seawall, some physical experiments are conducted $[5,6]$. Then a second-order time-domain model is applied to simulate this problem by Isaacson and Cheung [7]. Because wave interaction with the seawall usually occurs in a shallow water area, Boussinesq equation is widely used in this problem: Jamois et al. [8] modelled the action of 3D waves on a vertical seawall with the finite difference method; Fuhrman and Madsen [9] developed an interpolation technology in the Boussinesq model to capture the nonlinear free-surface elevation near a vertical wall; Chatjigeorgiou and Molin [10] investigated the wave action on a vertical seawall with a modified Boussinesq equation, where the third-order nonlinear wave effects were found. To better 
describe the nonlinearity during this process, the Green-Naghdi model [11] and fully nonlinear potential-flow theory [12] are also applied.

On the action of solitary waves on vertical seawalls, physical experiments are conducted [13]. The run-up of double solitary waves along a vertical wall is studied experimentally, which is found to be associated with the relative wave height and the distance between two solitary waves [14]. Based on low order expansion, the solitary wave run-up on a vertical seawall is studied with the third-order perturbation method by Su and Mirie [15] and spectral method by Fention and Rienecker [16]. For the advantages of nonlinearity, the boundary element method (BEM) is widely used in research about the solitary wave. Kim [17] simulated the solitary wave run-up on a vertical wall with BEM with fully-nonlinear free surface boundary conditions considered; Cooker et al. [18] simulated large-amplitude solitary waves acting on a vertical seawall problem with BEM, finding that the solitary wave can transfer to a series of dispersive waves after impacting the wall; Maiti and Sen [19] analyzed the solitary wave action on a vertical seawall using time-domain BEM, where waves acting on the vertical seawall showed stronger nonlinearity compared with those on an inclined wall. CFD is also used to study this problem, such as the method of smoothed particle hydrodynamics (SPH) [20].

Then, extending the simplified situation of continuous regular waves and solitary wave-to-wave train, some work has been done. Carbone et al. [21] observed in their numerical simulations that a multi-crest wave train can produce higher wave run-ups on a vertical wall, compared with the corresponding regular wave cases. The wave run-up can exceed the incident wave amplitude by a factor of five, which is a remarkable amplification of the incident wave amplitude. In their simulations, the Serre-Green-Naghdi (SGN) model, which satisfies a weak dispersion relationship, was adopted [22-25], which loses accuracy in water of finite depth for high amplitudes [26]. Akrish et al. [27] further investigated a modulated multi-crest wave train impacting a vertical seawall using a high-order spectral method (HOS). An unusual amplification of the wave run-up was also found, although the HOS model was still limited to the expansion of nonlinear terms in a weakly nonlinear sense. However, according to their studies, this process shows very strong nonlinearity. Some important phenomena may be missed due to the possible deficiency of nonlinearity. Thus, a model with enough nonlinearity should be applied. In this study, a higher-order BEM is applied which can preserve full nonlinearity. A numerical wave flume is developed to model the multi-crest wave train impacting on a vertical seawall. Strong nonlinear characteristics of wave run-ups and loads on the vertical seawall are further investigated.

\section{Materials and Methods}

Consider a 2D numerical wave flume with a constant depth $d$ and length $L$, as in Figure 1 . The left boundary is a wavemaker and the right boundary is a vertical seawall. The wavemaker, free surface, vertical seawall, seabed and fluid domain are denoted by $\Gamma_{w}, \Gamma_{f}, \Gamma_{b}, \Gamma_{d}$, and $V$, respectively. A Cartesian coordinate system is set in Figure 1, with the origin at the left side of the undisturbed free surface boundary, the $x$-axis pointing along the propagation direction of incoming waves, and the $z$-axis pointing upwards. The fluid is assumed to be incompressible, inviscid, and flow-irrotational. The velocity potential $\varphi(x, z, t)$ is introduced to describe the fluid motion.

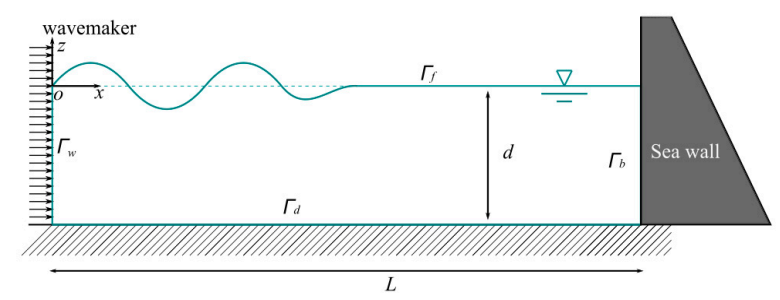

Figure 1. Sketch of the wave flume. 
The velocity potential $\varphi(x, z, t)$ satisfies Laplace equation in the fluid domain $V$

$$
\nabla^{2} \phi=0
$$

On the instantaneous free surface $\Gamma_{f}$, the kinematic and dynamic free-surface boundary conditions are satisfied as

$$
\begin{gathered}
\frac{\partial \eta}{\partial t}+\frac{\partial \phi}{\partial x} \frac{\partial \eta}{\partial x}=\frac{\partial \phi}{\partial z} \\
g \eta+\frac{\partial \phi}{\partial t}+(1 / 2)|\nabla \phi|^{2}=0
\end{gathered}
$$

where $g$ is the acceleration due to gravity and $\eta(x, t)$ is the free surface elevation.

On the seawall $\Gamma_{b}$ and seabed $\Gamma_{d}$, the impermeable boundary condition is imposed

$$
\partial \phi / \partial n=0
$$

where $n$ is the normal vector pointing to the outside of the tank.

On the wavemaker $\Gamma_{w}$, the boundary condition satisfies

$$
\partial \phi / \partial n=u(t)
$$

where $u(t)$ is the horizontal velocity of the wavemaker. The initial wave condition is given as

$$
\phi=0, \eta=0
$$

The pressure can be obtained according to Bernoulli equation. The horizontal wave load on the vertical seawall can be calculated through an integration of the pressure as follows

$$
f=\int_{\Gamma_{b}} p d z=-\rho \int_{\Gamma_{b}}\left(\phi_{t}+g z+\frac{1}{2}\left|\nabla \phi^{2}\right|\right) d z
$$

where $\varphi_{t}$ is the time derivatives of $\varphi$. It can be derived by solving the boundary value problem

$$
\begin{gathered}
\nabla^{2} \phi_{t}=0 \text { in } V \\
\phi_{t}=-g \eta-(1 / 2)|\nabla \phi|^{2} \text { on } \Gamma_{f} \\
\partial \phi_{t} / \partial n=0 \text { on } \Gamma_{b} \text { and on } \Gamma_{d} \\
\partial \phi_{t} / \partial n=\dot{u}(t) \text { on } \Gamma_{w}
\end{gathered}
$$

where $\dot{u}(t)$ is the horizontal acceleration of the wavemaker.

Based on the second Green's theorem, Equations (1) and (8) can be transferred into the boundary integral equation as follows [28]

$$
C(\boldsymbol{p}) \psi(\boldsymbol{p}, t)=\int_{\Gamma}\left[\psi(\boldsymbol{q}, t) \frac{\partial G(\boldsymbol{q}, \boldsymbol{p})}{\partial n}-G(\boldsymbol{q}, \boldsymbol{p}) \frac{\partial \psi(\boldsymbol{q}, t)}{\partial n}\right] d \Gamma
$$

where $\psi$ represents the velocity potential $\phi$ and the time derivative of potential $\phi_{t}$ corresponding to Equations (1) and (8), respectively. $\boldsymbol{p}\left(x_{0}, z_{0}\right)$ is collocation point, $\boldsymbol{q}(x, z)$ is a point on the boundaries, $C$ is the solid angle coefficient and is solved by the indirect method [29], $\Gamma$ denotes the whole fluid boundary, and $G$ is the simple Green function

$$
G(p, q)=\ln |p-q|
$$


where $|p-q|$ represents the distance between the two points. Then, the boundary surface is discretized into quadratic curve elements. The location and velocity potential on each element can be obtained through the interpolation

$$
S(\xi)=\sum_{n=1}^{M} h_{n}(\xi) S_{n}
$$

where $M$ is the number of nodes on each element, $S$ represents physical quantities including $x, z, \varphi$ and $\partial \phi / \partial n, \xi \in[-1.0,1.0]$ is a local intrinsic coordinate, and $h_{n}$ is the shape function at the $n$th node

$$
\left\{\begin{array}{l}
h_{1}(\xi)=\xi(\xi-1) / 2 \\
h_{2}(\xi)=(1+\xi)(1-\xi) \\
h_{3}(\xi)=\xi(1+\xi) / 2
\end{array}\right.
$$

The integral equation is formulated in the following form after discretization:

$$
\begin{aligned}
& -C(p) \psi(p) \\
& +\sum_{e=1}^{N e} \sum_{m=1}^{M} \int_{-1}^{1}\left[\frac{\partial G(p, q(\xi))}{\partial n} \cdot h_{m}(\xi) \cdot J(\xi)\right] d \xi \cdot \psi_{d(e, m)} \\
& =\sum_{e=1}^{N e} \sum_{m=1}^{M} \int_{-1}^{1}\left[G(\boldsymbol{p}, \boldsymbol{q}(\xi)) \cdot h_{m}(\xi) \cdot J(\xi)\right] d \xi \cdot\left(\frac{\partial \psi}{\partial n}\right)_{d(e, m)}
\end{aligned}
$$

where $d(e, m)$ is the serial number of the $m$ th node on the eth element, $N_{e}$ is the amount of the elements and $J(\xi)$ is the Jacobian determinant which can be written as

$$
|J(\xi)|=\sqrt{(\partial x / \partial \xi)^{2}+(\partial z / \partial \xi)^{2}}
$$

At every time step, the velocity potential on the free surface and the normal derivative of the velocity potential on solid boundaries are known. The velocity potential on $\Gamma_{w}, \Gamma_{b}$, and $\Gamma_{d}$, and its normal derivative on $\Gamma_{f}$ can be obtained by solving Equation (16). Spatial derivatives of the velocity potential can be computed through a numerical differential. The time derivatives of the velocity potential and wave elevation can be computed according to free-surface boundary conditions. The fourth-order Runge-Kutta method is adopted to update the position and velocity potential of the free surface to the next time step [29].

\section{Results and Discussion}

In this section, single-, double- and multi-crest wave trains acting on the vertical seawall are simulated. For the single- and double-crest cases, to avoid reflection at the wavemaker, the flume length $L$ and the total time $T_{f}$ for computation are set as

$$
\begin{gathered}
L=\left(N_{w}+0.5\right) \lambda \\
T_{f}=N_{w} \cdot T+\frac{L}{\sqrt{g(d+a)}}
\end{gathered}
$$

where $N_{w}$ is the number of waves generated in the flume and $\lambda$ is the wave length. The free-surface boundary is discretized by 400 elements per wavelength, while the wavemaker and seawall are by 10 elements, respectively. When the $\omega$ is small, the wave period gets longer, and a smaller time step is needed. In order to guarantee an accurate result, the time step is set to $\Delta t=T / 400$ with some allowance, where $T$ is the wave period, as shown in Figure 2. The static water depth and gravitational acceleration are used as the bases of the dimensionalization. All variables are nondimensionalized. To be specific, the distance, velocity and time can be normalized by $d, \sqrt{g d}$, and $\sqrt{d / g}$, respectively. 


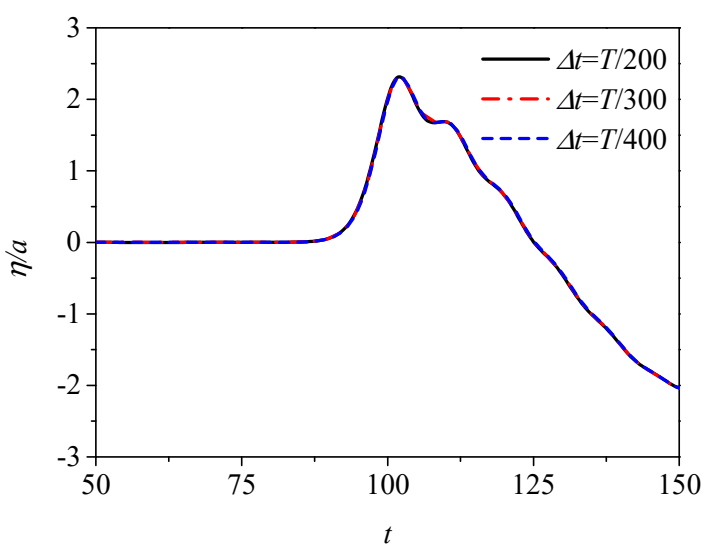

Figure 2. Convergence test for $a=0.05, \omega=0.1$.

\subsection{Single-Crest Wave Train}

For the case of a single-crest wave train, the velocity of the wavemaker $u(t)$ is defined by

$$
\begin{gathered}
u(t)=\frac{\eta_{0}(t) c_{s}}{d+\eta_{0}(t)} \\
\eta_{0}(t)=a \sin (\omega t) H(T-t) \\
c_{s}=\sqrt{g(d+a)}
\end{gathered}
$$

where $a$ and $\omega$ are the amplitude and angular frequency of waves, respectively. $H(t)$ is the Heaviside function and $C_{s}$ is the wave speed

$$
H(t)=\{0, t<0 ; 1, t>0\}
$$

We set the angular frequency as $\omega=0.145$ and amplitude as $a=0.05$. Figure 3 shows the time history of the free-surface run-up along the vertical seawall. Figure 4 gives the profile of free surface at $t=1.67 \mathrm{~T}$. Figure 5 further compares the maximum wave run-up (MWR) along the vertical seawall for more wave frequencies. The obtained numerical results are compared with those of the SGN model [21]. In all these figures, the numerical results agree well with the corresponding SGN results, as a verification of the present model. The MWR is approximately twice the amplitude of incident waves when the wave frequency is low, which is consistent with the result predicted by linear potential-flow theory. At $\omega=\omega_{0}=0.145$, the MWR reaches its maximum of 2.3a, indicating strong nonlinear characteristics. Here, $\omega_{0}$ represents the frequency at which the MWR is the largest.

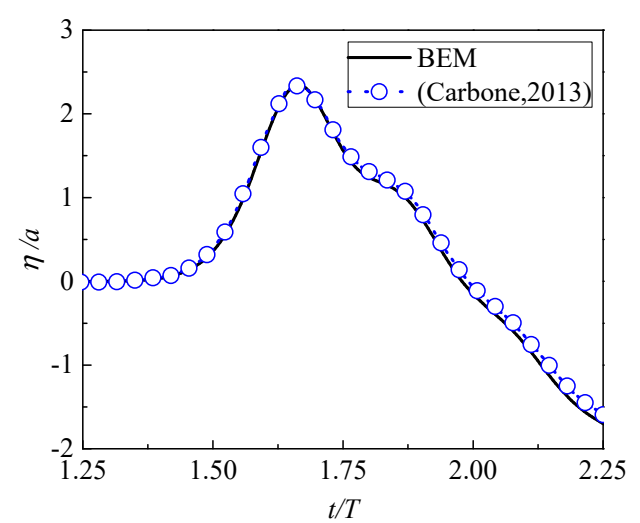

Figure 3. History of wave run-up along the vertical seawall for the single-crest wave train case. 


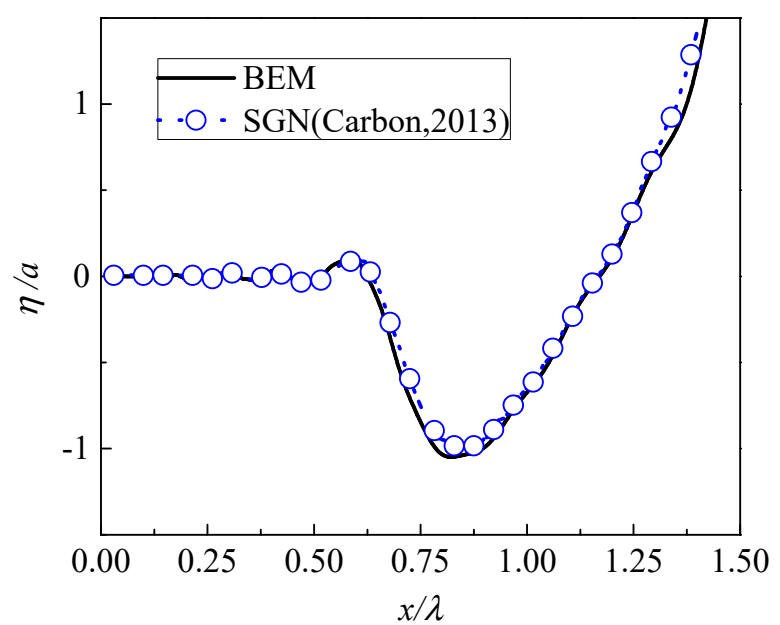

Figure 4. Free surface profile at $t=1.67 T$ for the single-crest wave train case.

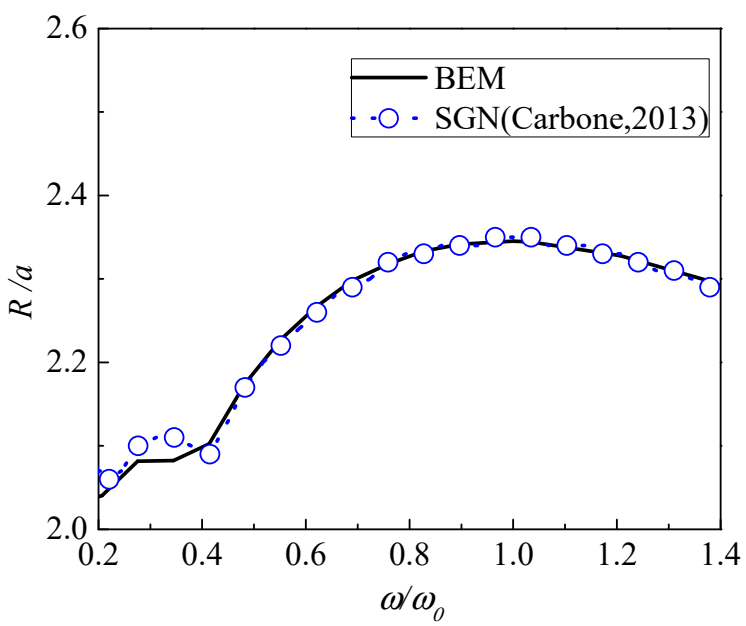

Figure 5. MWR along the vertical seawall of different frequencies.

\subsection{Double-Crest Wave Train}

This subsection further considers cases of the double-crest wave train. The incident wave frequency is set as $\omega=\omega_{0}=0.02$. Three different amplitudes of incident waves are considered in Figure 6. The present results agree well with the SGN model results when the first wave crest impacts on the wall. However, the discrepancies between the two models become evident during the run-up process of the second wave crest. The wave run-up history of the double-crest wave train shows a feature of high-frequency oscillations. The oscillation is more obvious during the run-up process of the second wave crest. This shows strong nonlinear and high-frequency characteristics, which may be due to the interaction between components of difference frequencies. For the case of $a=0.05$, the MWR of the present model can reach 4.4 times the incident wave amplitude, while it is 3.8 times for the SGN model. Then we decrease the nonlinearity by using a smaller incident wave amplitude. We can find, after decreasing nonlinearity, the second run-up histories match well, for $a=0.0479$. Although the amplitudes of these cases are close, they can still lead to obvious differences.

Then, we compare the MWR results for more wave frequencies. The MWR along the vertical seawall for double-crest wave trains of different frequencies and amplitudes is shown in Figure 7. The MWR in the present model gets closer to the SGN model at a higher wave frequency. For a larger wave amplitude case of $a=0.05$, the difference between the results of two models is more evident. The MWR from the present model can reach 5 times the incident wave amplitude, while it is 3.8 times 
for the SGN model. The difference between the two models is due to the limitation of the SGN model which only satisfies the weakly dispersion relationship based on the shallow water assumption. The SGN model will lose accuracy in water of finite depth for high amplitudes [26]. For large wave amplitude cases, the double-crest wave train shows stronger wave nonlinearity, which can be reflected by the present numerical model. In other words, the SGN model actually underestimates the MWR.

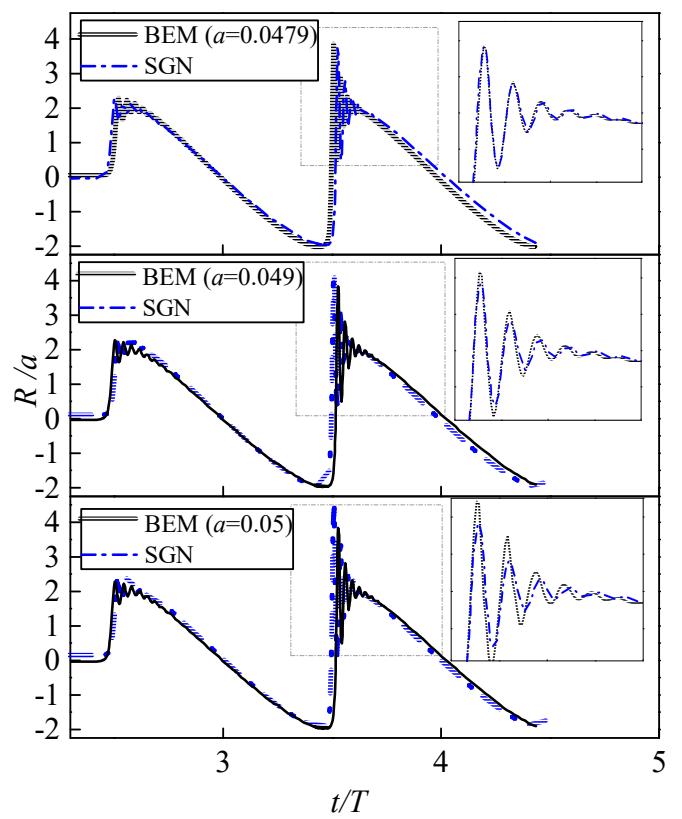

Figure 6. History of wave run-up along the vertical seawall for double-crest wave train cases.

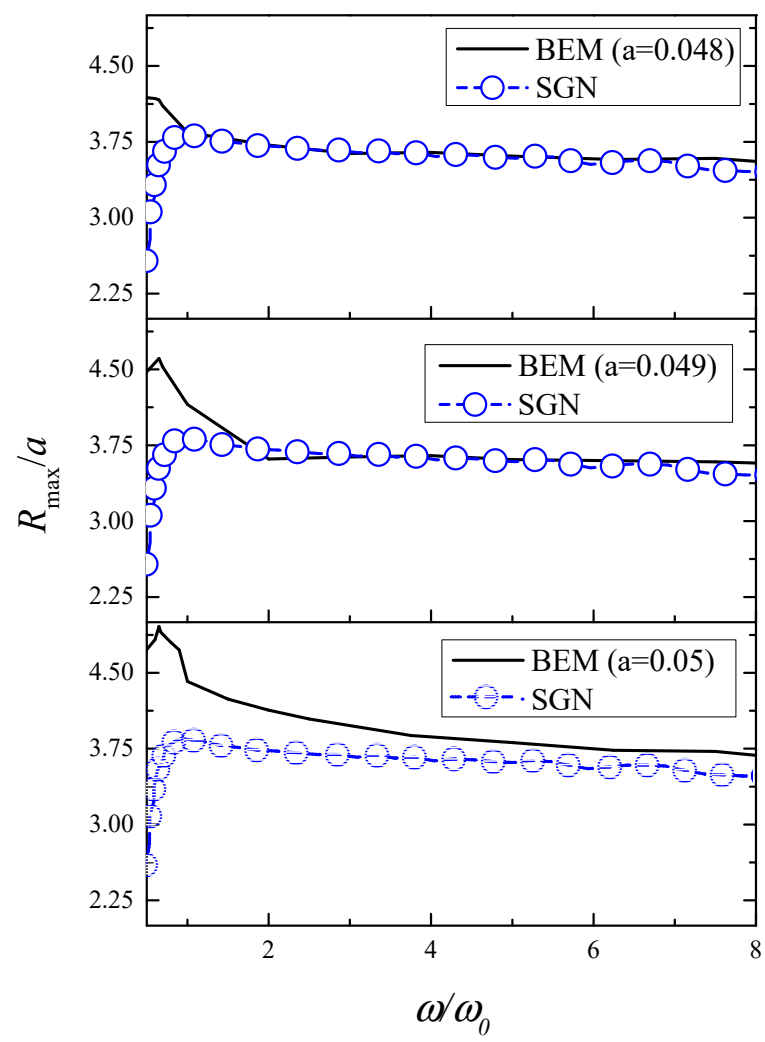

Figure 7. MWR along the vertical seawall for double-crest wave train cases. 
Nonlinear analysis is further conducted for the case of $a=0.05$. Histories of the wave run-up along the vertical seawall for double-crest wave trains of different wave frequencies $(\omega=0.02, \omega=0.1$ and $\omega=0.2$ ) are shown in Figure 8. Linear results obtained by linearizing the nonlinear free surface Equations (2) and (3) are given for comparison. The nonlinear results are evidently different from the corresponding linear results. It shows that the linear results essentially underestimate the maximum wave run-up along the seawall. Meanwhile, the nonlinear results show high-frequency oscillations which cannot be found in the linear results. This means the oscillation is due to the nonlinear effect. The oscillation becomes weaker, and the MWR gets smaller with the increase of the incident wave frequency, which are both typical nonlinear characteristics. In other words, the nonlinear effect produced by the wave-wave interaction becomes weaker when the frequency is high. Evolution of wave profiles for the case of double-crest waves with $\omega=0.2$ is given in Figure 9. During the wave impact process, the second wave crest first meets head-on the reflected wave crest of the first wave. Then, two wave crests merge into one single-crest wave, whose amplitude is about twice that of the incident wave. After that, two wave crests separate and continue propagating along their original direction. During this process, free-surface oscillation occurs between the two crests. When the second crest reaches the vertical seawall after the interaction with the reflected wave, it begins climbing up. The run-up on the wall also has the characteristics of high-frequency oscillation. However, the MWR is much bigger than twice the incident wave amplitude. Histories of wave force acting on the vertical seawall for the case of double-crest waves of different wave frequencies are shown in Figure 10. The nonlinear characteristic is similar to the wave run-up process in Figure 8.

The MWR along the vertical seawall and the maximum wave force acting on the vertical seawall for double-crest wave trains of different wave frequencies are shown in Figure 11. The trends of these two physical quantities are very similar. The amplitude peaks locate at $\omega=0.2$. The MWR is 5 times the incident wave amplitude, and the maximum wave force can reach 2.5 times that of the linear theory one. For $\omega>\omega_{0}$, both of the wave run-up and wave force of the nonlinear waves reduce, but they are still larger than the corresponding linear results.

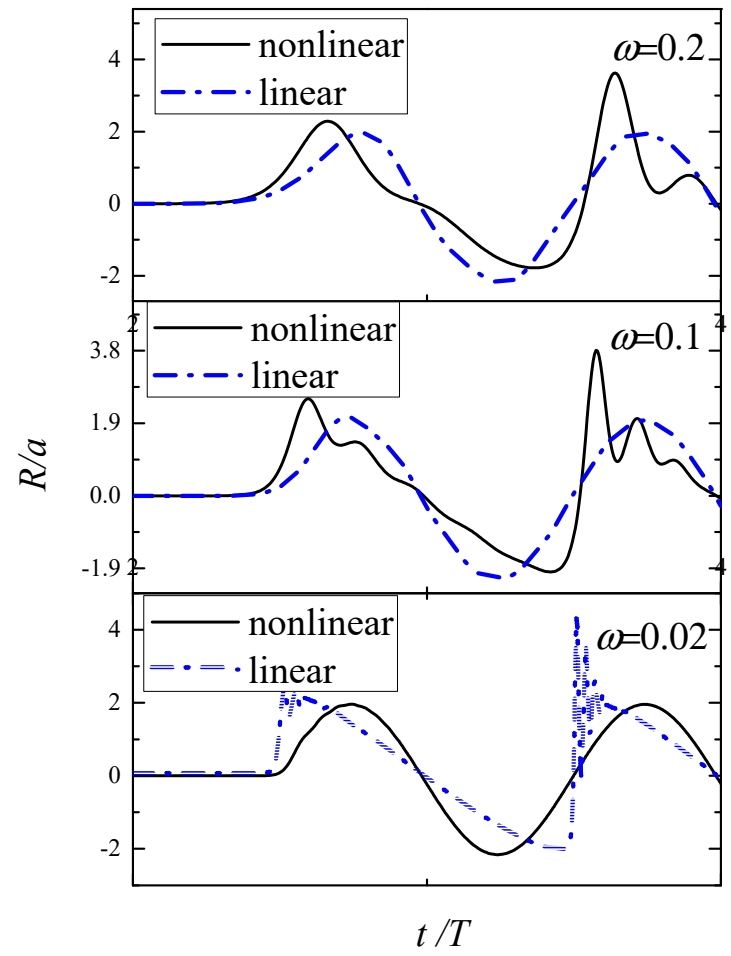

Figure 8. History of wave run-up along the vertical seawall for the case of double-crest wave train of different wave frequencies. 


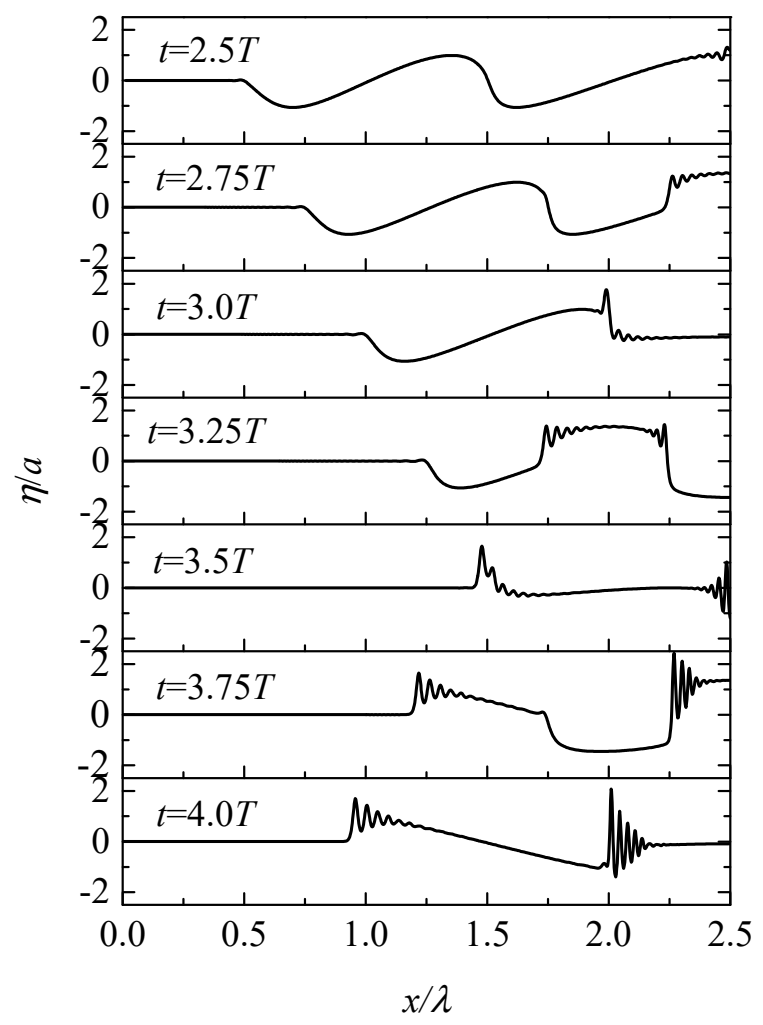

Figure 9. Evolution of wave profiles for the case of double-crest wave train.

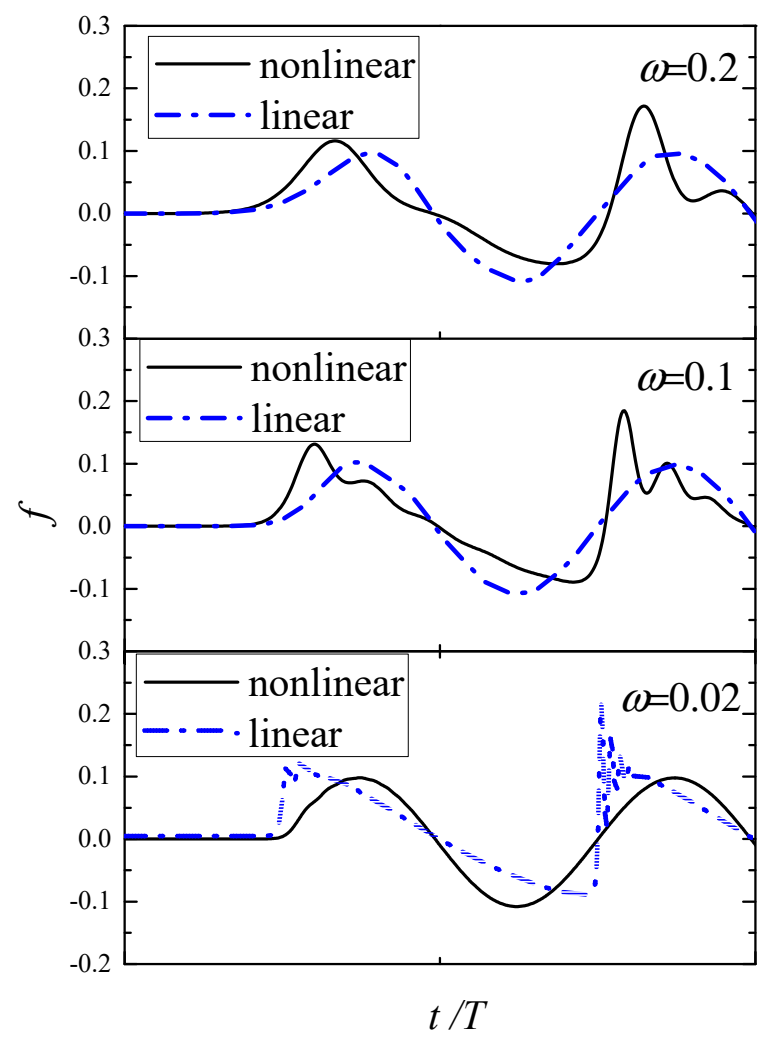

Figure 10. History of wave force acting on the vertical seawall for double-crest wave train of different wave frequencies. 


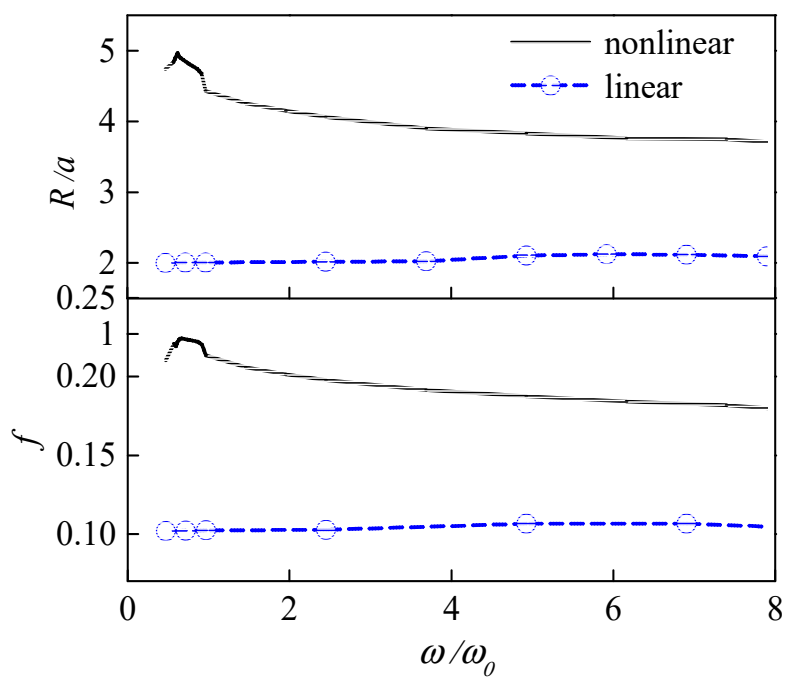

Figure 11. MWR along the vertical seawall and maximum wave force acting on the vertical seawall for double incident waves of different wave frequencies.

Spectra of the wave run-up and wave force histories on the vertical seawall for the double-crest wave train at $\omega_{0}$ are shown in Figure 12. The wave component at $\omega_{0}$ dominates both in the linear and nonlinear results. Different from the linear results, the nonlinear results also have the peaks appearing at $\omega=2 \omega_{0}, \omega=3 \omega_{0}, \omega=4 \omega_{0}$, etc. This suggests that there is an energy transfer among wave components of different frequencies during the wave-wave interaction. Due to the nonlinearity, the energy of the wave component at the fundamental frequency is transferred into higher-frequency wave components and leads to the amplification of run-up as well as strong oscillation. The run-up and wave force of the double-crest wave train on the vertical seawall has typical nonlinear characteristics.

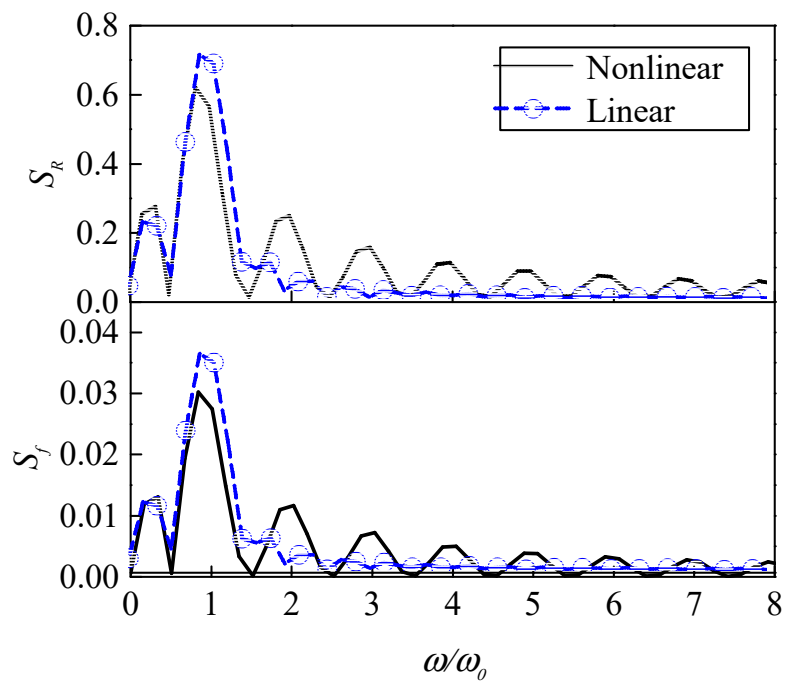

Figure 12. Spectra ofthe wave run-up and the wave force on the vertical seawall.

\subsection{Multi-Crest Wave Train}

In this section, the multi-crest wave train is further considered. A typical multi-crest wave train can be generated in the numerical wave flume under the following wavemaker excitation [27] 


$$
\begin{gathered}
S(t)=S_{0} \exp \left(-\left(\frac{t-\tau}{\mu T}\right)^{2}\right) \cos (\omega(t-\tau)) \\
S_{0}=a \frac{\sinh 2 k+2 k}{2(\cosh 2 k-1)}
\end{gathered}
$$

where $S(t)$ is the horizontal displacement of the wavemaker, $k$ is the wavenumber, $\mu$ is the parameter that controls the width of the waves envelope, $\tau$ is the parameter that controls the initiation of the wavemaker's motion to avoid an abrupt disturbance, and $S_{0}$ is the stroke of the wavemaker which is relevant to the maximum incident wave amplitude. Wave run-up histories on the wall are presented in Figure 13. For all cases, non-dimensional parameters are set as $a=0.05, \mu=2.0$ and $\tau=5 T$. From the wavemaker motion Equation (23) we can expect that the wave amplitude can get larger as time goes on before reaching its maximum at $t=\tau$.

In Figure 13, the run-up amplification and oscillation mentioned in Figure 8 also appear in both cases. The maximum wave run-up reaches $R_{\max }=6.2 a$ for case 1 , and $R_{\max }=5.6 a$ for case 2 . It shows that the amplitude amplification effect of the waves is even stronger than that of the double-crest wave train cases.

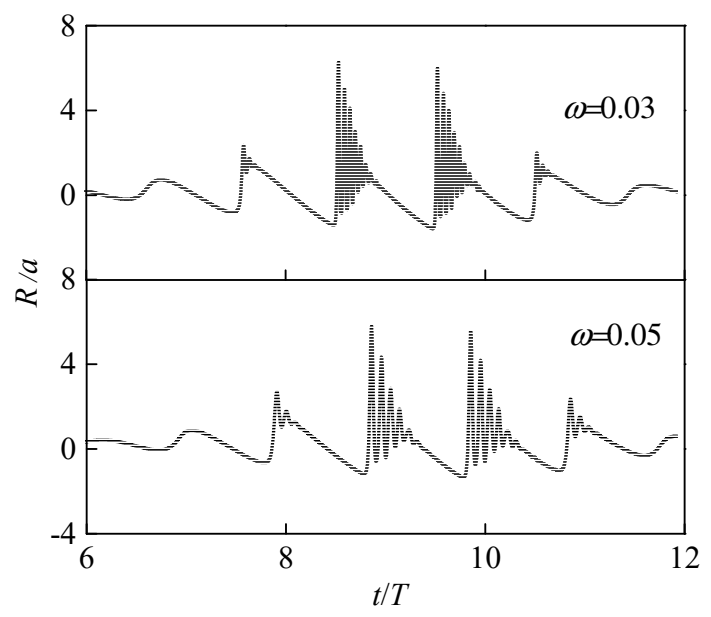

Figure 13. History of wave run-up along the vertical wall for multi-crest wave train cases of different wave frequencies.

The MWR along the vertical wall for multi-crest wave trains of different wave frequencies is shown in Figure 14. For convenience, the length of the wave flume is chosen as 4 times the wave length. Similarly, there is a tendency that MWR goes down with increasing the incident wave frequency, which is similar to double-crest wave train cases. By applying the Fast Fourier Transform, the amplitude spectra of the run-up histories in Figure 13 can be obtained in Figure 15. $\omega^{\prime}$ denotes the frequencies of each component. It is found that the amplitude of the wave component at the fundamental frequency is dominant. The amplitude of higher-order wave components gradually reduces.

The length scale of the multi-crest wave train is further investigated by changing the value of $\mu$. The wave run-ups corresponding to $\mu=2.0$ and $\mu=4.0$ along the vertical seawall are shown in Figure 16. At the two sides of the wave train, the wave amplitudes of the incident waves are relatively small, compared to that in the middle. This wave train may be more common than single and double-crest waves in real states of the sea. In Figure 16, we can find that the wave amplitude in the middle is amplified significantly due to the higher nonlinearity. When increasing the length of the wave train, more large-amplitude wave peaks appear, although the largest wave run-up along the seawall does not change much with increasing $\mu$. The longer the wave train, the more high-amplitude crests exist. 


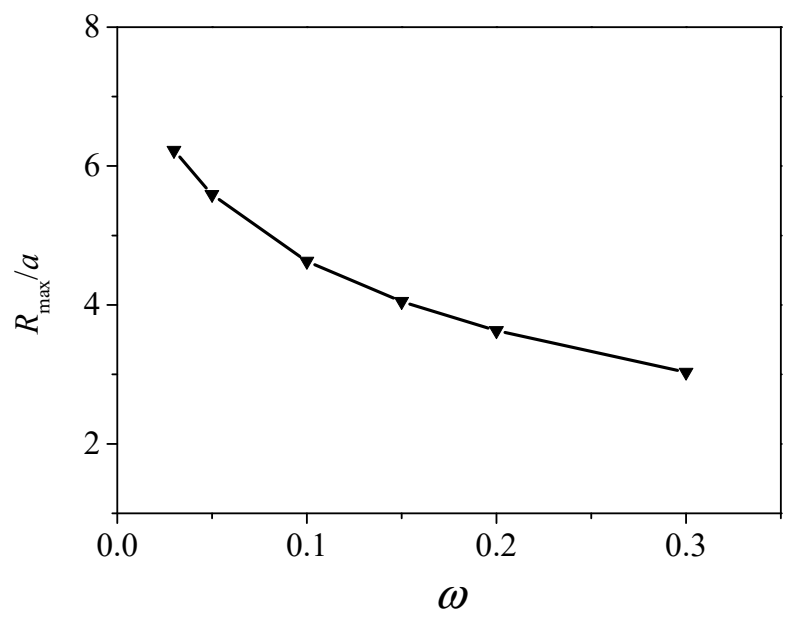

Figure 14. MWR along the vertical wall for multi-crest wave train cases of different wave frequencies.

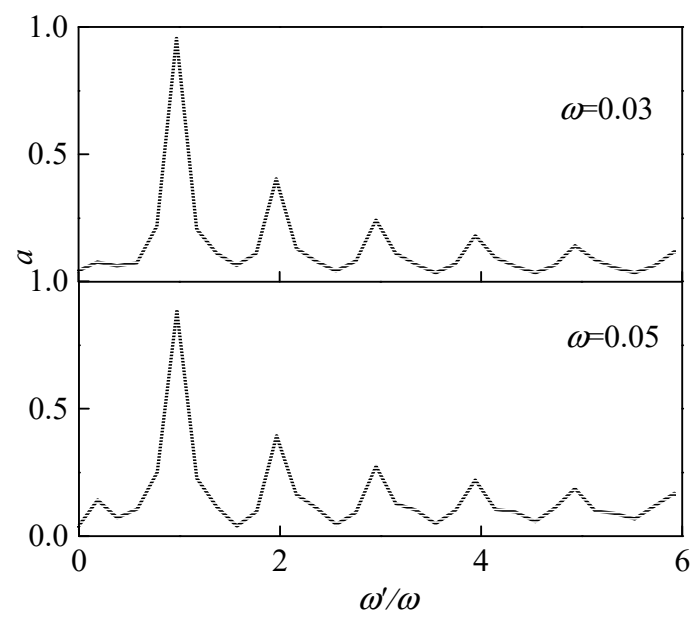

Figure 15. Spectra of wave run-up on a vertical seawall for multi-crest wave train cases of different wave frequencies.

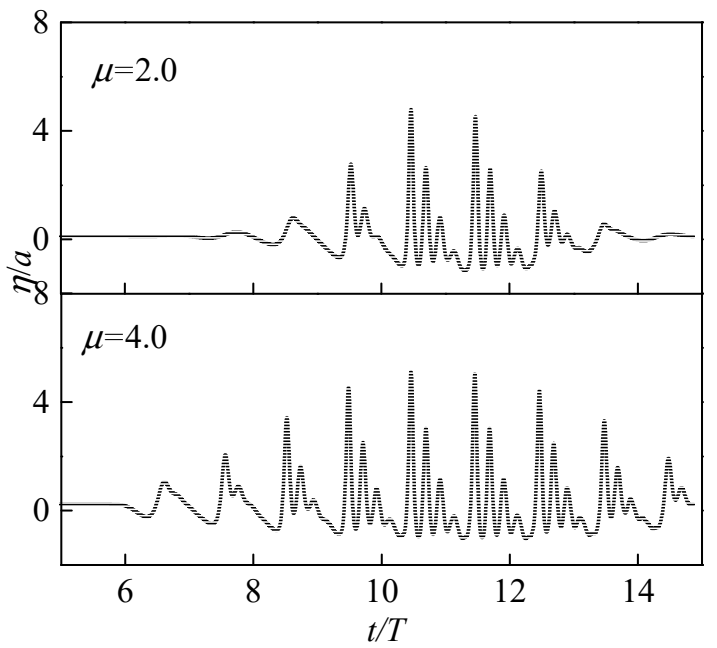

Figure 16. History of wave run-up along the vertical wall for multi-crest wave train cases of different wave frequencies. 


\section{Conclusions}

A 2D fully-nonlinear numerical wave flume is developed based on the time-domain higher-order boundary element method. Wave train impact on a vertical seawall is investigated. Cases of the single-, double- and multi-crest wave trains are discussed. The phenomena observed in this study indicate that multi-crest wave trains can lead to an extraordinarily high wave run-up along the vertical seawall, which should attract people's attention in coastal engineering designs. Some specific findings are summarized as follows. For single-crest wave train cases, the present nonlinear results are compared with the solution of the SGN theory, showing a good agreement. For double-crest wave train cases, the SGN model generally underestimates the maximum wave run-up (MWR) along the vertical seawall. Nonlinear analysis for the double-crest cases is further carried out. Compared with the linear results, the nonlinear results show larger wave run-ups and higher-frequency oscillations. The higher-frequency oscillation weakens for higher-frequency incident wave trains. The wave load shows similar nonlinear behavior as the wave run-up. Through an FFT analysis of the wave run-up and wave force histories, it is found that the wave component at the fundamental frequency is transformed to higher-frequency wave components, showing a typical nonlinear characteristic. For the multi-crest wave train cases, unusual run-up can be observed. In some cases, the wave run-up along the vertical seawall can reach about 6 times that of the incident waves. In this run-up process, high-frequency oscillations associated with the wave impact process can also be observed, which may also endanger coastal structures. It should be pointed out that experiments are still required in the future to further confirm the above findings obtained from numerical simulations.

Author Contributions: Numerical simulations and result analyses were conducted by D.N., X.L. and C.Z. All authors reviewed the manuscript.

Funding: National Key R\&D Program of China (Grant No. 2016YFE0200100), National Science Foundation of China (Grant Nos. 51679036 and 51709038), and China Postdoctoral Science Foundation (No. 2018M630289).

Conflicts of Interest: The authors declare no conflict of interest.

\section{References}

1. Onorato, M.; Osborne, A.R.; Serio, M.; Bertone, S. Freak waves in random oceanic sea states. Phys. Rev. Lett. 2001, 86, 5831-5834. [CrossRef] [PubMed]

2. Hattori, M.; Arami, A.; Yui, T. Wave impact pressure on vertical walls under breaking waves of various types. Coast. Eng. 1994, 22, 79-114. [CrossRef]

3. Kirkgöz, M.S. An experimental investigation of a vertical wall response to breaking wave impact. Ocean Eng. 1990, 17, 379-391. [CrossRef]

4. Grue, J.; Pelinovsky, E.N.; Fructus, D.; Talipova, T.; Kharif, C. Formation of undular bores and solitary waves in the strait of malacca caused by the 26 December 2004 Indian ocean tsunami. J. Geophys. Res. Oceans 2008, 113, C05008. [CrossRef]

5. Mallayachari, V.; Sundar, V. Standing wave pressures due to regular and random waves on a vertical wall. Ocean Eng. 1995, 22, 859-879. [CrossRef]

6. Tsai, C.P.; Lin, Y.J.; Hung, C.W. Examination of Wave Pressures on Breakwater over Steep Beach; International Society of Offshore and Polar Engineers: Osaka, Japan, 2009; pp. 1064-1068.

7. Isaacson, M.; Cheung, K.F. Second order wave diffraction around two-dimensional bodies by time-domain method. Appl. Ocean Res. 1991, 13, 175-186. [CrossRef]

8. Jamois, E.; Fuhrman, D.R.; Bingham, H.B.; Molin, B. A numerical study of nonlinear wave run-up on a vertical plate. Coast. Eng. 2006, 53, 929-945. [CrossRef]

9. Fuhrman, D.R.; Madsen, P.A. Simulation of nonlinear wave run-up with a high-order boussinesq model. Coast. Eng. 2008, 55, 139-154. [CrossRef]

10. Chatjigeorgiou, I.K.; Molin, B. Third-order interactions, wave run-up and hydrodynamic loading on a vertical plate in an infinite wave field. Appl. Ocean Res. 2013, 41, 57-64. [CrossRef]

11. Kim, J.W.; Ertekin, R.C. A numerical study of nonlinear wave interaction in regular and irregular seas: Irrotational green-naghdi model. Mar. Struct. 2000, 13, 331-347. [CrossRef] 
12. Grilli, S.T.; Guyenne, P.; Dias, F. A fully non-linear model for three-dimensional overturning waves over an arbitrary bottom. Int. J. Numer. Methods Fluids 2015, 35, 829-867. [CrossRef]

13. Liu, P.L.F.; Albanaa, K. Solitary wave runup and force on a vertical barrier. J. Fluid Mech. 2004, 505, $225-233$. [CrossRef]

14. Xuan, R.T.; Wei, W.U.; Hua, L.; Fang, Y.L. An experimental study on run-up of double solitary waves against a vertical wall. Chin. J. Hydrodyn. 2013, 28, 241-251.

15. Su, C.H.; Mirie, R.M. On head-on collision between two solitary-waves. J. Fluid Mech. 2008, 98, 509-525. [CrossRef]

16. Fenton, J.D.; Rienecker, M.M. A fourier method for solving nonlinear water-wave problems: Application to solitary-wave interactions. J. Fluid Mech. 2006, 118, 411-443. [CrossRef]

17. Kim, S.K.; Liu, L.F.; Liggett, J.A. Boundary integral equation solutions for solitary wave generation, propagation and run-up. Coast. Eng. 1983, 7, 299-317. [CrossRef]

18. Cooker, M.J.; Weidman, P.D.; Bale, D.S. Reflection of a high-amplitude solitary wave at a vertical wall. J. Fluid Mech. 1997, 342, 141-158. [CrossRef]

19. Maiti, S.; Sen, D. Computation of solitary waves during propagation and runup on a slope. Ocean Eng. 1999, 26, 1063-1083. [CrossRef]

20. Jian, W.; Sim, S.Y.; Huang, Z.; Lo, E.Y.-M. Modelling of solitary wave run-up on an onshore coastal cliff by smoothed particle hydrodynamics method. Procedia Eng. 2015, 116, 88-96. [CrossRef]

21. Carbone, F.; Dutykh, D.; Dudley, J.M.; Dias, F. Extreme wave runup on a vertical cliff. Geophys. Res. Lett. 2013, 40, 3138-3143. [CrossRef]

22. Green, A.E.; Laws, N.; Naghdi, P.M. On the theory of water waves. Proc. R. Soc. Lond. A 1974, 338, 43-55. [CrossRef]

23. Green, A.E.; Naghdi, P.M. A derivation of equations for wave propagation in water of variable depth. J. Fluid Mech. 2006, 78, 237-246. [CrossRef]

24. Zhao, B.B.; Ertekin, R.C.; Duan, W.Y. A comparative study of diffraction of shallow-water waves by high-level ign and gn equations. J. Comput. Phys. 2015, 283, 129-147. [CrossRef]

25. Zhao, B.B.; Duan, W.Y.; Ertekin, R.C. Application of higher-level gn theory to some wave transformation problems. Coast. Eng. 2014, 83, 177-189. [CrossRef]

26. Li, Y.A.; Hyman, J.M.; Choi, W. A numerical study of the exact evolution equations for surface waves in water of finite depth. Stud. Appl. Math. 2010, 113, 303-324. [CrossRef]

27. Akrish, G.; Schwartz, R.; Rabinovitch, O.; Agnon, Y. Impact of extreme waves on a vertical wall. Nat. Hazards 2016, 84, 637-653. [CrossRef]

28. Ning, D.Z.; Teng, B.; Zhao, H.T.; Hao, C.L. A comparison of two methods for calculating solid angle coefficients in a biem numerical wave tank. Eng. Anal. Bound. Elem. 2010, 34, 92-96. [CrossRef]

29. Ning, D.Z.; Zhuo, X.L.; Teng, B. Focused waves interaction with a vertical wall. In Proceedings of the Twenty-third International Offshore and Polar Engineering Conference; International Society of Offshore and Polar Engineers, Anchorage, AK, USA, 30 June-5 July 2013.

(C) 2018 by the authors. Licensee MDPI, Basel, Switzerland. This article is an open access article distributed under the terms and conditions of the Creative Commons Attribution (CC BY) license (http:/ / creativecommons.org/licenses/by/4.0/). 